\section{Restauration des voies corticales lésées par greffe de neurones}

Nissrine Ballout, Sophie Péron, Afsaneh Gaillard

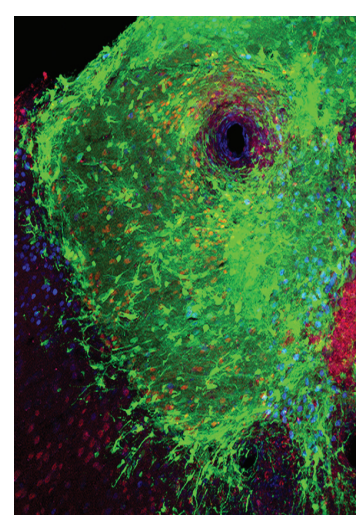

Laboratoire des neurosciences expérimentales et cliniques, Inserm U1084, université de Poitiers, 1, rue Georges Bonnet, 86073 Poitiers, France. afsaneh.gaillard@ univ-poitiers.fr

\section{Transplantation dans le cortex immature}

Des études menées chez le rat ont montré que les neurones corticaux immatures greffés dans le cortex moteur de rats nouveau-nés sont capables d'émettre des projections vers les régions du cerveau qui sont normalement connectées avec le cortex moteur, comme le striatum ou le thalamus [10] et la moelle épinière [11]. Les neurones greffés sont également aptes à recevoir des afférences spécifiques du thalamus et du cortex, montrant leurs intégrations anatomiques dans les circuits moteurs de l'hôte [12].

Dans le cadre de la réparation des voies corticales par greffe de neurones, un facteur important à prendre en considération est l'origine des neurones corticaux qui sont greffés. II a été démontré que la réparation des circuits corticaux endommagés n'est possible que lorsque les neurones greffés sont de la même origine que la région corticale lésée. En effet, seuls les neurones du cortex moteur restaurent les projections motrices endommagées, contrairement à des neurones provenant du cortex visuel et greffés dans cette région [13, 14]. Ainsi, l'origine des neurones corticaux greffés apparaît comme un élément déterminant pour la mise en place des circuits neuronaux qui seront néoformés. En termes de fonctionnalité, les transplants homotopiques (c'est-à-dire provenant du cortex moteur et greffés dans le cortex moteur) peuvent être bénéfiques, ce qui n'est pas le cas des transplants hétérotopiques (cortex visuel greffé dans le cortex moteur), dont l'effet peut même être délétère [15].

Des tests comportementaux ont permis de démontrer les effets bénéfiques, sur le plan fonctionnel, des greffes de cortex embryonnaire d'origine moteur transplantées dans le cortex moteur de rats nouveaux-né et lésés [10]. La greffe semble en effet favoriser durablement la récupération des capacités motrices altérées par la lésion, 
et, inversement, l'ablation du greffon conduit à des déficits moteurs semblables à ceux qui sont observés après lésion corticale [16]. L'intégration fonctionnelle des transplants est également démontrée par leur capacité à répondre à une stimulation. L'enregistrement en électrophysiologie de l'activité unitaire des transplants a en effet permis de montrer que les neurones greffés peuvent répondre à une stimulation sensorielle périphérique (appliquée sur les pattes antérieures de l'animal), ou à une stimulation électrique de régions projetant vers la greffe, comme le thalamus [17].

\section{Transplantation dans le cerveau adulte}

Les résultats qui concernent la transplantation de neurones immatures dans le cortex lésé chez l'adulte restent controversés. En effet, une absence de projections des neurones immatures greffés dans le cortex adulte lésé, ou dans les cas les plus favorables, des projections éparses et à très faible distance du transplant ont été observées $[18,19]$. Dans les études que nous avons relatées, les connections entre l'hôte et le transplant ont été révélées par injection de traceurs rétrogrades ou antérogrades et l'identification des transplants était réalisée par marquage au moment de la transplantation (par exemple avec des particules d'or [13]). Ces techniques présentaient des limites pour l'identification précise et complète du transplant et de ses projections. Afin de surmonter ces limites, la greffe chez des souris sauvages d'explants d'embryons exprimant de manière ubiquitaire la GFP (green fluorescent protein), une protéine fluorescente verte, sous le contrôle du promoteur de l'actine- $\beta$, est apparue être un outil pertinent pour révéler, de manière indiscutable, le transplant et l'ensemble des projections qu'il développe [20]. Les travaux réalisés dans notre laboratoire [21] ont ainsi permis une avancée majeure dans l'étude de la reconstruction des voies lésées dans le cerveau mature. Dans cette étude, le cortex moteur de souris adultes sauvages a été lésé. Un fragment de cortex embryonnaire d'origine moteur exprimant la GFP, prélevé aux $14^{\mathrm{e}}$ jours embryonnaire ( $\left.\varepsilon 14\right)$, a été placé dans la cavité lésionnelle du cortex de la souris adulte. Après 8 semaines, les projections émises par le transplant ont été analysées. II a pu être observé que les neurones du transplant envoyaient des projections vers les cibles corticales et sous-corticales appropriées de l'hôte, y compris à longue distance, jusqu'à la moelle épinière. Ces projections respectaient l'organisation topographique des projections corticales : une partie des fibres étaient myélinisées, et des contacts synaptiques réciproques s'étaient établis entre l'hôte et le transplant. Ce travail a donc mis en évidence la permissivité à la repousse axonale du cerveau mature, et la possibilité de reconstruire point-à-point les voies motrices lésées chez l'adulte par transplantation de neurones corticaux embryonnaires.

Les neurones corticaux transplantés induisent une récupération motrice [9]. Ils répondent électrophysiologiquement ou métaboliquement à une stimulation sensorielle ou électrique de l'hôte [6-22] ou lors de l'exécution d'une tâche motrice [3] et présentent une activité de décharge spontanée [23].

\section{Composition cellulaire et organisation cytoarchitecturale des transplants}

À la suite de ces observations, une question restait à résoudre. En effet, si les neurones transplantés sont effectivement capables de développer des projections respectant une organisation topographique normale, la composition cellulaire et l'organisation cytoarchitecturale du tissu greffé sont-elles semblables à celles du cortex en conditions normales?

Le cortex cérébral est formé d'une centaine de types de neurones différents organisés en six couches cellulaires et de nombreuses études ont révélé une perte de l'organisation laminaire normale dans les transplants. La distribution cellulaire y est en effet soit diffuse, soit organisée en bandes, ou en amas (clusters) séparés par des faisceaux de fibres $[6,10,24,25]$. Ces amas cellulaires expriment néanmoins les marqueurs des couches corticales, comme Cuxl (cut-like homeobox 1) pour les couches superficielles, et Ctip2 (chicken ovalbumin upstream promoter transcription factor-interacting protein 2, ou BCL1lb [B-cell lymphoma/leukemia 11b]) et Foxp2 (forkhead box $P 2$ ) pour les couches profondes (Figure 1) [7].

Les neurones corticaux sont subdivisés en deux classes principales: les neurones à projections excitatrices, glutamatergiques, représentant $80 \%$ des neurones du cortex, et les interneurones inhibiteurs, constituant environ $20 \%$ des neurones restants. Une question majeure, au regard de l'efficacité des transplants à réparer les circuits corticaux lésés, est la capacité des cellules embryonnaires transplantées à se différencier vers le phénotype neuronal approprié. Si l'étude des connexions mises en place par les neurones transplantés met en évidence la présence de neurones à projections dont les afférences sont spécifiques, les données de la littérature présentent peu d'informations quant à la composition cellulaire des transplants de fragments embryonnaires corticaux. On retrouve, dans les greffes, des cellules pyramidales, non-pyramidales, et gliales [26]. Des arguments penchent en faveur d'une organisation cellulaire conforme à celle du cortex normal : le glutamate s'avère être, par électrophysiologie, le principal neurotransmetteur excitateur dans ce modèle de transplantation [6], et des neurones de type GABAergique [6], ainsi que des récepteurs glutamatergiques, cholinergiques et GABAergiques, sont révélés par immunohistochimie [6]. En revanche, dans certains cas, la densité cellulaire dans les transplants serait globalement supérieure à celle de l'hôte [27], et la densité relative des neurones GABAergiques serait réduite [27]. 


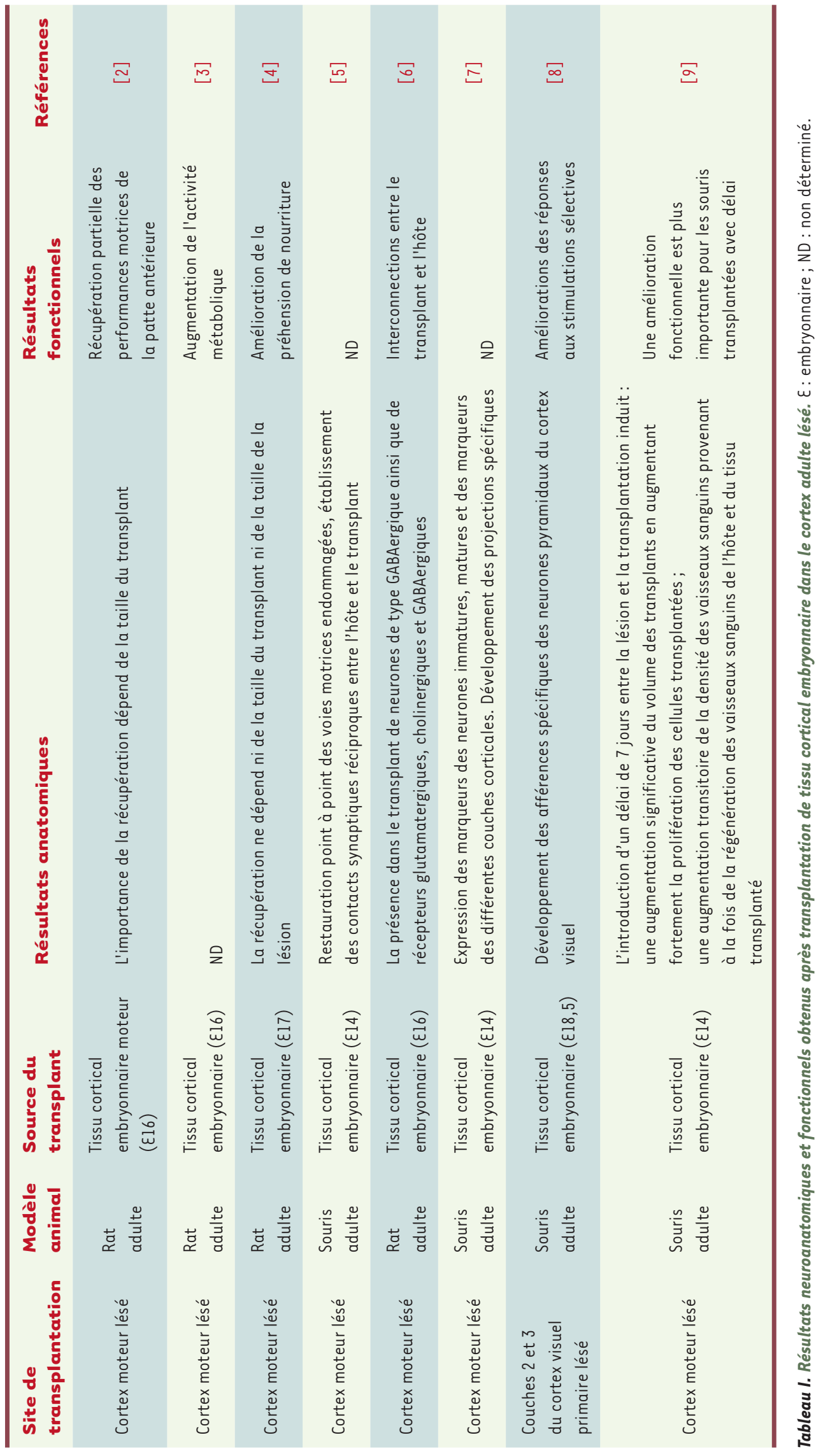



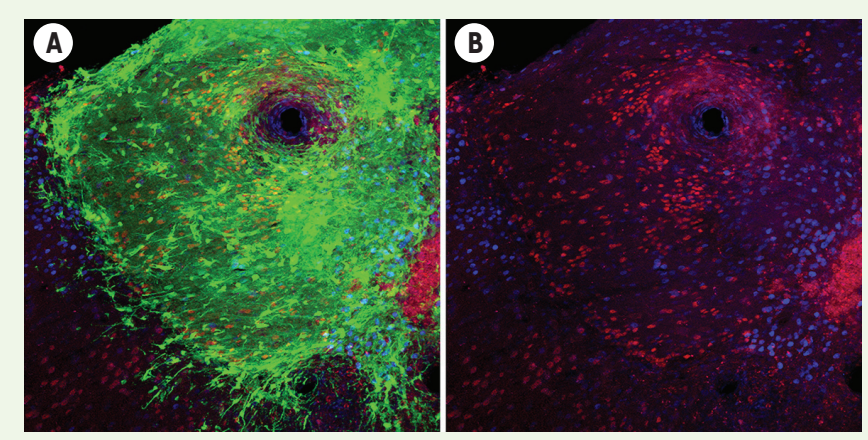

Figure 1. Expression de marqueurs de couches corticales par les neurones greffés. $(A, B)$ Immunomarquage illustrant le transplant (en vert), Cuxl (cut-like homeobox 1), un marqueur exprimé par les neurones des couches superficielles (en bleu) et CTIP2 (chicken ovalbumin upstream promoter transcription factor-interacting protein), un marqueur spécifique des neurones des couches profondes (en rouge)

\section{Les facteurs influençant le développement des neurones transplantés}

L'efficacité de la transplantation dépend du niveau de survie du transplant, de sa capacité à se différencier selon le phénotype neuronal approprié et à restaurer les projections spécifiques. Plusieurs facteurs influencent la survie et le développement des cellules greffées. Afin d'optimiser le potentiel thérapeutique de la transplantation neuronale, il est donc important de définir des conditions expérimentales qui soient optimales.

\section{Âge du donneur/âge du receveur}

Les neurones corticaux semblant présenter le plus haut potentiel de croissance sont ceux prélevés entre $\varepsilon 14$ et $\varepsilon 15$ chez la souris [28], ce qui correspond au pic de neurogenèse des neurones à projections corticales. La plupart des études de transplantation sont réalisées chez le nouveau-né ou le jeune adulte; peu d'entre elles ont examiné la réceptivité du cerveau âgé à ces transplants. Pourtant, les processus de plasticité, de régénération, et de repousse axonale sont influencés par le niveau de maturité du tissu receveur. Pour certains auteurs, passée la période périnatale, l'âge du receveur ne semble plus avoir d'influence [29]. Cependant, dans des modèles de transplantation de neurones corticaux [30] ou hippocampiques [31], les niveaux de survie, de croissance et de récupération anatomique et fonctionnelle sont moindres chez la souris âgée (après 12 mois).

\section{Présence ou absence de lésion}

De nombreux modèles animaux de lésion corticale sont utilisés afin de mimer les différents types que l'on observe chez l'homme. Citons, parmi ceux-ci, le modèle de lésion ischémique, obtenu par l'occlusion de l'artère cérébrale moyenne, les modèles de lésion cérébrale traumatique induite par un impact localisé, le modèle neurotoxique, obtenu par administration d'un composé neurotoxique, et le modèle de lésion corticale par aspiration, que nous avons utilisé dans nos études. Ce modèle permet de cibler une zone précise du cortex. L'aspiration entraîne une destruction immédiate des corps cellulaires et des axones afférents et efférents.

Des études réalisées il y a une vingtaine d'années avaient montré que l'absence de lésion précédant la transplantation corticale chez le rat nouveau-né ou adulte semblait limiter la croissance du transplant et le développement de ses projections [32]. Ces résultats ont été confirmés plus récemment chez la souris adulte, montrant que l'absence de lésion ne permet pas aux neurones corticaux transplantés de développer des projections, y compris vers les cibles proches comme le cortex ipsilatéral [33]. Ces observations peuvent s'expliquer par l'absence de voies en dégénérescence laissées vacantes chez l'hôte après lésion. En effet, les fibres du transplant semblent préférentiellement emprunter les voies en dégénérescence pour étendre leurs projections. La réaction astrocytaire post-lésionnelle semble également participer à ce processus : après lésion, les astrocytes réactifs de l'hôte auraient la capacité de ré-exprimer des facteurs trophiques (normalement exprimés pendant le développement) et guideraient les fibres développées par le transplant [33]. Une régénération appropriée par les neurones transplantés, dans le cerveau mature, dépendrait donc en partie des facteurs exprimés le long des voies lésées.

\section{Neuroinflammation}

La neuroinflammation est un processus complexe caractérisé par l'activation des cellules gliales, la libération de facteurs pro- ou anti-inflammatoires, et le recrutement de cellules immunitaires dans le cerveau. L'inflammation peut à la fois être bénéfique ou néfaste en fonction du temps, après la lésion. L'inflammation post-traumatique peux influencer la survie des neurones greffés ainsi que le développement de projections issues de ces neurones. Inversement, les cellules transplantées peuvent moduler l'inflammation post-traumatique.

Les greffes de neurones immatures survivent relativement bien dans un cerveau mature [34]. Cependant, une réaction inflammatoire survient à la suite de toutes lésions, induisant l'activation de médiateurs cellulaires responsables de la neuro-inflammation, principalement produits par les cellules gliales [35]. La microglie, les astrocytes et les oligodendrocytes sont recrutés et activés au site de la lésion. En conditions physiologiques, l'accès des cellules périphériques au système nerveux est étroitement contrôlé par la barrière hématoencéphalique $(B H \varepsilon)$. Lors d'une lésion associée à 
une rupture de la $B H \varepsilon$, différents types cellulaires circulants peuvent migrer du sang vers le site de la lésion, sous l'effet de différentes cytokines et chimiokines. Les neutrophiles sont les premiers à traverser la BHE, suivis des monocytes et des lymphocytes [36].

De nombreuses études se sont intéressées à la neuroinflammation, pour son rôle aussi bien délétère que bénéfique sur les neurones [37]. Une double fonction, neuroprotectrice et neurotoxique, est couramment attribuée à la microglie. L'activation soutenue des cellules microgliales conduit en effet à la production de cytokines pro-inflammatoires et d'espèces réactives oxygénées et azotées, qui permettent de lutter contre les agents pathogènes environnants. Cette activation peut ainsi se prolonger, se propager et amplifier la destruction des neurones. Parallèlement, un rôle protecteur de la microglie sur les cellules environnantes a été suggéré par leur capacité à sécréter des cytokines anti-inflammatoires et des molécules neurotrophiques qui participent à la survie neuronale et à la réparation tissulaire, comme le NGF (nerve growth factor), la NT3 (neurotrophine 3) et le BDNF (brain-derived neurotrophic factor).

\section{Facteurs trophiques}

De nombreux facteurs sont libérés par les neurones, les cellules immunitaires et les cellules gliales, au niveau du site des lésions à des fins de protection et pour favoriser la survie cellulaire et la réparation des voies lésées. Après lésion corticale, par exemple, une augmentation des niveaux d'expression du GMFB (glia maturation factor beta) autour du site de la lésion a ainsi été rapportée [38]. Le GMFB est produit par les astrocytes en réaction à la lésion. II induit la sécrétion de facteurs neurotrophiques, comme le BDNF et le NGF [38]. Les niveaux d'expression de FGF (fibroblast growth factor) [39] et de GDNF (glial cell linederived neurotrophic factor) [39] sont également augmentés.

Combiner l'administration de facteurs trophiques à la transplantation pourrait favoriser la survie des cellules greffées. Dans une série de travaux, Lee et al. $[40,41]$ ont réalisé, après lésion ischémique, des expériences de transplantation dans le cortex de cellules souches neurales humaines (hNSC) sur-exprimant le GDNF ou le BDNF. Les cellules transplantées se différencient alors en neurones, et leur survie et les améliorations fonctionnelles qu'elles induisent sont augmentées par rapport à des cellules souches neurales humaines qui ne sur-expriment pas ces facteurs.

\section{Délai entre lésion et transplantation neuronale}

Introduire un délai entre la lésion et la transplantation favoriserait la survie et le développement du transplant [32, 42, 43]. Dans un modèle de lésion ischémique par occlusion de l'artère cérébrale moyenne (MCAO), Grabowski et al. ont rapporté un effet similaire pour des délais de transplantation de $5-7$ jours ou de 8 semaines avec un délai le plus favorable pour 3 semaines. D'autres études indiquent que des délais courts seraient préférables : l'effet serait en effet optimal lorsque le délai coïncide avec la libération maximale de facteurs neurotrophiques par les astrocytes autour de la lésion, soit 9 jours dans le modèle de transplantation de tissu embryonnaire striatal dans le cortex entorhinal [44]. De même, la récupération fonctionnelle à des tests de mémorisation spatiale, après lésion du cortex frontal médian, est meilleure lorsque la transplantation est réalisée 7 ou 14 jours après la lésion, les transplantations réalisées 30 ou 60 jours après la lésion n'ayant pas d'effet [45].

L'hypothèse que l'introduction d'un délai entre lésion et transplantation permettrait la néoformation de vaisseaux, la libération par les astrocytes de facteurs trophiques autour de la lésion et dans la cavité lésionnelle, et la diminution des niveaux de neurotoxines produits [42] a été formulée. L'introduction d'un délai d'une semaine entre la lésion et la transplantation corticale est en effet bénéfique d'un point de vue anatomique et fonctionnel [9]. Introduire un délai entre la lésion du cortex moteur et la transplantation du cortex moteur embryonnaire augmente significativement le volume des transplants en favorisant fortement la prolifération des cellules transplantées. L'analyse de la vascularisation des transplants montre également une augmentation transitoire de la densité des vaisseaux sanguins lorsqu'un délai est imposé entre la lésion et la transplantation, avec une différence d'origine des vaisseaux sanguins colonisant le transplant. En effet, lorsque la transplantation est réalisée immédiatement après la lésion, ceux-ci proviennent presque exclusivement de l'hôte. En revanche, lorsqu'un délai est introduit entre la lésion et la transplantation, les vaisseaux proviennent à la fois de la régénération des vaisseaux sanguins de l'hôte et du tissu transplanté (Figure 2).

L'introduction d'un délai entre la lésion et la transplantation n'altère pas la distribution des projections développées par les cellules transplantées. En revanche, le nombre de projections émises par les neurones transplantés vers toutes les cibles corticales appropriées du cortex moteur est augmenté lorsqu'un délai est introduit entre la lésion et la transplantation (Figure 2).

D'un point de vue fonctionnel, les résultats que nous avons obtenus montrent un déficit significatif des capacités motrices des souris lésées par rapport aux souris contrôles. Les souris transplantées sans délai présentent une tendance à l'amélioration qui n'est cependant pas significative par rapport à des souris contrôles. L'amélioration fonctionnelle est en revanche notable et significative pour les souris qui ont été transplantées avec un délai.

\section{Vascularisation des neurones transplantés}

Néo-angiogenèse (extension de l'arbre vasculaire à partir de vaisseaux préexistants) et néo-vasculogenèse (formation de novo de vaisseaux sanguins) sont des processus qui sont induits par des facteurs comme le VEGF (vascular endothelial growth factor), le FGF et 


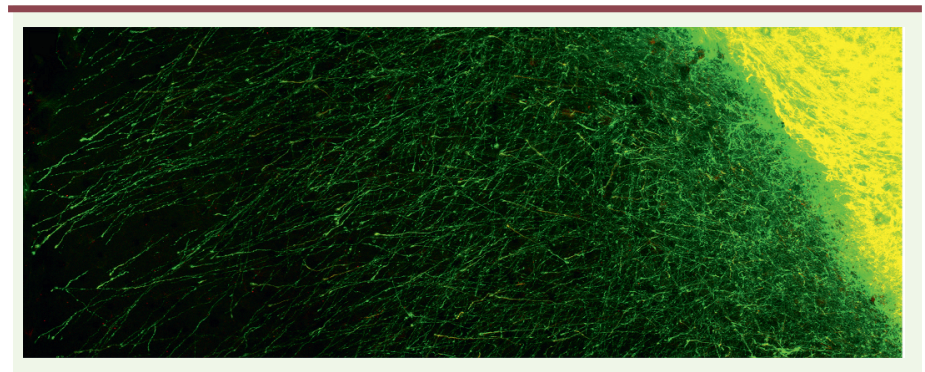

Figure 2. Projections développées par les neurones greffés. Fibres GFP (green fluorescent protein) quittant le transplant 7 jours après la transplantation. Une partie des fibres GFP (en vert) expriment la doublecortine (marquage rouge surimposé à la GFP et apparaissant en jaune), un marqueur exprimé par les neurones immatures en développement.

les angiopoïétines. Dans le modèle de lésion de la moelle épinière, la néo-angiogenèse post-traumatique est un phénomène dynamique et transitoire qui présente un pic d'intensité une semaine après la lésion. Parallèlement, la repousse axonale est plus importante et plus rapide à proximité des vaisseaux sanguins [46].

L'importance de la vascularisation des transplants, qui permet un apport en oxygène et en composés métaboliques, a été pressentie dès les travaux de Dunn, en 1917 [47], et largement confirmée depuis. Les vaisseaux sanguins présents dans les transplants proviennent de l'hôte et du greffon et l'interaction entre les cellules endothéliales de l'hôte et du transplant est primordiale pour l'établissement d'un réseau vasculaire fonctionnel. La survie du tissu greffé dépend initialement de l'apport sanguin qu'il reçoit du tissu hôte. Les cellules endothéliales du transplant participent ensuite à la vascularisation et la formation d'anastomoses permet de réunir les vaisseaux des deux origines [48]. Les données temporelles concernant la vascularisation des transplants sont variables d'une étude à l'autre, notamment, selon que la transplantation ait été réalisée sous forme de bloc tissulaire ou de suspension cellulaire $[49,50]$. Savoir si la densité du réseau vasculaire du transplant est comparable à celle de l'hôte [23, 48] ou moindre [50] reste également à déterminer.

\section{Conclusion}

La transplantation de neurones embryonnaires permet de comprendre des mécanismes fondamentaux liés au développement et à la réparation cérébrale. Elle a permis de mettre en évidence le potentiel, longtemps sous-estimé (notamment chez l'adulte), des thérapies cellulaires dans le cadre de lésions du système nerveux central. Des études cliniques utilisant des cellules embryonnaires neuronales réalisées, en particulier, chez des patients parkinsoniens, mettent en évidence un effet bénéfique de ces greffes chez certains de ces patients. Cependant, l'origine fœtale des cellules utilisées pour la transplantation soulève des problèmes éthiques et logistiques. Pour ces raisons, des sources alternatives de neurones ont été envisagées et, ces dernières années, les études de thérapie cellulaire fondées sur l'utilisation de cellules souches, ou plus récemment de cellules pluripotentes induites
(IPSC pour induced pluripotent stem cells) se sont multipliées, permettant de surmonter les limites posées par l'utilisation des cellules embryonnaires. $\diamond$

\section{SUMMARY}

Restoration of damaged cortical pathways by neural grafting

The motor cortex plays a central role in the control, planning, and execution of voluntary motor commands in mammals. The loss of cortical neurons is a common feature of many neuropathological conditions such as traumatic and ischemic lesions or several neurodegenerative diseases. Cell transplantation presents a promising therapeutic strategy to overcome the limited abilities of axonal regrowth and spontaneous regeneration of the adult central nervous system. In this review, we will present a historical review of brain transplantation and the current state of research in the field of cortical transplantation. $\diamond$

\section{LIENS D'INTÉRÊT}

Les auteurs déclarent n'avoir aucun lien d'intérêt concernant les données publiées dans cet article.

\section{RÉFÉRENCES}

1. Darling WG, Pizzimenti MA, Morecraft RJ. Functional recovery following motor cortex lesions in non-human primates: experimental implications for human stroke patients. J Integr Neurosci 2011 ; 10 : 353-84.

2. Plumet J, Ebrahimi A, Guitet J, et al. Partial recovery of skilled forelimb reaching after transplantation of fetal cortical tissue in adult rats with motor cortex lesion - anatomical and functional aspects. Restor Neurol Neurosci $1993: 6: 9-27$.

3. Ebrahimi-Gaillard A, Beck T, Gaillard F, et al. Transplants of embryonic cortical tissue placed in the previously damaged frontal cortex of adult rats : local cerebral glucose utilization following execution of forelimb movements. Neuroscience $1995: 64:$ 49-60.

4. Riolobos AS, Heredia M, de la Fuente JA, et al. Functional recovery of skilled forelimb use in rats obliged to use the impaired limb after grafting of the frontal cortex lesion with homotopic fetal cortex. Neurobiol Learn Mem $2001: 75: 274-92$

5. Gaillard A, Prestoz L, Dumartin B, et al. Reestablishment of damaged adult motor pathways by grafted embryonic cortical neurons. Nat Neurosci 2007 ; $10: 1294-9$.

6. Santos-Torres J, Heredia M, Riolobos AS, et al. Electrophysiological and synaptic characterization of transplanted neurons in adult rat motor cortex. J Neurotrauma $2009 ; 26$ : 1593-607.

7. Ballout N, Frappé I, Péron S, et al. Development and maturation of cortical embryonic neurons grafted into the damaged adult motor cortex. Front Neural Circuits $2016 ; 10: 55$.

8. Falkner S, Grade S, Dimou L, et al. Transplanted embryonic neurons integrate into adult neocortical circuits. Nature $2016: 539: 248-53$

9. Péron S, Droguerre M, Debarbieux F, et al. A delay between lesion and transplantation enhances graft integration and ameliorates repair and recovery. J Neurosci 2017 ; 37 : 1820-34

10. Plumet J, Cadusseau J, Roger M. Fetal cortical transplants reduce motor deficits resulting from neonatal damage to the rat's frontal cortex. Neurosci Lett $1990 ; 109: 102-6$.

11. Floeter $M$, Jones $\varepsilon$. Connections made by transplants to the cerebral cortex of rat brains damaged in utero. J Neurosci $1984 ; 4: 141-50$.

12. Frappé $I$, Roger M, Gaillard A. Transplants of fetal frontal cortex grafted into the occipital cortex of newborn rats receive a substantial thalamic input from nuclei normally projecting to the frontal cortex. Neuroscience $1999 ; 89: 409-21$. 


\section{RÉFÉRENCES}

13. Ebrahimi-Gaillard A, Guitet J, Garnier C, et al. Topographic distribution of efferent fibers originating from homotopic or heterotopic transplants: heterotopically transplanted neurons retain some of the developmental characteristics corresponding to their site of origin. Dev Brain Res $1994 ; 77: 271-83$.

14. Gaillard A, Gaillard F, Roger M. Neocortical grafting to newborn and adult rats: developmental, anatomical and functional aspects. Adv Anat Embryol Cell Biol 1998 ; 148 : 1-86.

15. Barth TM, Stanfield BB. Homotopic, but not heterotopic, fetal cortical transplants can result in functional sparing following neonatal damage to the frontal cortex in rats. Cereb Cortex $1994 ; 4$ 271-8.

16. Sandor R, Gonzalez MF, Moseley M, et al. Motor deficits are produced by removing some cortical transplants grafted into injured sensorimotor cortex of neonatal rats. J Neural Transplant Plast $1991 ; 2: 221-33$.

17. Neafsey $\varepsilon$ J, Sørensen JC, Tønder $N$, et al. Fetal cortical transplants into neonatal rats respond to thalamic and peripheral stimulation in the adult. An electrophysiological study of single-unit activity. Brain Res $1989 ; 493: 33-40$

18. Guitet J, Garnier C, Ebrahimi-Gaillard A, et al. Efferents of frontal or occipital cortex grafted into adult rat's motor cortex. Neurosci Lett $1994 ; 180: 265-8$.

19. Sorensen JC, Grabowski M, Zimmer J, et al. Fetal neocortical tissue blocks implanted in brain infarcts of adult rats interconnect with the host brain. Exp Neurol 1996; $138: 227-35$.

20. Gaillard F, Domballe L, Gaillard A. Fetal cortical allografts project massively through the adult cortex. Neuroscience $2004 ; 126: 631-7$

21. Gaillard F, Jaber M. Repair of damaged adult cortex by transplantation of embryonic neurons. Med Sci $2008: 24: 132-4$

22. Grabowski M, Brundin $P$, Johansson BB. Functional integration of cortical grafts placed in brain infarcts of rats. Ann Neurol $1993 ; 34: 362-8$

23. Senatorov VV, Obuhova GP, Fülöp Z. Electrophysiological and morphological properties of embryonic neocortical grafts developing in different regions of the host rat brain. J Neura Transplant Plast $1991 ; 2$ : 125-40.

24. Pinaudeau C, Gaillard A, Roger M. Stage of specification of the spinal cord and tectal projections from cortical grafts. Eur J Neurosci $2000 ; 12: 2$ 486-96.

25. Gaillard A, Roger M. Early commitment of embryonic neocortical cells to develop area-specific thalamic connections. Cereb Cortex $2000 ; 10: 443-53$

26. Belichenko PV, Mattsson B, Johansson BB. Neuronal and fibre organization in neocortical graft placed in post-ischaemic adult rat brain: a three-dimensional confocal microscopy study. J Comp Pathol $2001 ; 124: 142-8$

27. Bragin A, Takács J, Vinogradova 0, et al. Age-related loss of GABA-positive and GABA-negative neurons in neocortical transplants. J Neural Transplant Plast $1993 ; 4: 53-9$.

28. Das GD, Hallas BH, Das KG. Transplantation of brain tissue in the brain of rat. I. Growth characteristics of neocortical transplants from embryos of different ages. Am J Anat $1980 ; 158$ : 135-45.

29. Hallas BH, Das GD, Das KG. Transplantation of brain tissue in the brain of rats. II. Growth characteristics of neocortical transplants in hosts of different ages. Am J Anat $1980 ; 158$ : 147-59.

30. Eriksdotter-Nilsson M, Olson L. Growth of brain tissue grafts is dependent upon host age. Mech Ageing Dev $1989 ; 49$ : 1-22.

31. Zaman V, Shetty AK. Combined neurotrophic supplementation and caspase inhibition enhances survival of fetal hippocampal CA3 cell grafts in lesioned CA3 region of the aging hippocampus. Neuroscience $2002 ; 109$ : 537-53.

32. Gibbs RB , Cotman CW. Factors affecting survival and outgrowth from transplants of entorhinal cortex. Neurosci $1987 ; 21: 699-706$.
33. Gaillard A, Jaber M. Is the outgrowth of transplant-derived axons guided by host astrocytes and myelin loss? Cell Adh Migr 2007 ; 1 : 161-4.

34. Bjorklund A, Stenevi U, Dunnett SB, et al. Cross-species neural grafting in a rat model of Parkinson's disease. Nature $1982 ; 298: 652-4$.

35. Russo MV, McGavern DB. Inflammatory neuroprotection following traumatic brain injury. Science $2016 ; 353: 783-5$.

36. Kato H, Walz W. The initiation of the microglial response. Brain Pathol 2000 $10: 137-43$.

37. Block ML, Zecca L, Hong JS. Microglia-mediated neurotoxicity: uncovering the molecular mechanisms. Nat Rev Neurosci $2007 ; 8$ : 57-69.

38. Hotta N, Aoyama M, Inagaki M, et al. Expression of glia maturation factor beta after cryogenic brain injury. Mol Brain Res 2005 ; 133 : 71-7.

39. Cheng $\mathrm{Q}$, Di Liberto V, Caniglia G, et al. Time-course of GDNF and its receptor expression after brain injury in the rat. Neurosci Lett $2008 ; 439: 24-9$.

40. Lee HJ, Park IH, Kim HJ, et al. Human neural stem cells overexpressing glial cell line-derived neurotrophic factor in experimental cerebral hemorrhage. Gene Ther 2009; 16 : 1066-76.

41. Lee HJ, Lim IJ, Lee MC, et al. Human neural stem cells genetically modified to overexpress brain-derived neurotrophic factor promote functional recovery and neuroprotection in a mouse stroke model. J Neurosci Res 2010 ; 88 : 3282-94.

42. Gonzalez M, Sharp F. Fetal frontal cortex transplanted to injured motor/ sensory cortex of adult rats. I. NADPH-diaphorase neurons. J Neurosci 1987 ; $7: 2991-3001$.

43. Grabowski M, Johansson BB, Brundin P. Survival of fetal neocortical grafts implanted in brain infarcts of adult rats: the influence of postlesion time and age of donor tissue. Exp Neurol 1994: 127 : 126-36.

44. Nieto-Sampedro M, Manthrope M, Barbin G, Varon S, Cotman CW. Injury induced neuronotrophic activity in adult rat brain: correlation with survival of delayed implants in the wound cavity. J Neurosci $1983 ; 3: 2219-29$.

45. Stein DG, Palatucci C, Kahn D, et al. Temporal factors influence recovery of function after embryonic brain tissue transplants in adult rats with frontal cortex lesions. Behav Neurosci $1988 ; 102: 260-7$

46. Dray C, Rougon G, Debarbieux F. Quantitative analysis by in vivo imaging of the dynamics of vascular and axonal networks in injured mouse spinal cord. Proc Natl Acad Sci USA 2009; 106 : 9459-64.

47. Dunn $\varepsilon H$. Primary and secondary findings in a series of attempts to transplant cerebral cortex in the albino rat. J Comp Neurol $1917 ; 27$ : 56582.

48. Krum JM, Rosenstein JM. Patterns of angiogenesis in neural transplant model: II. Fetal neocortical transplants. J Comp Neurol 1988 ; 271 : 331-45.

49. Dusart I, Nothias F, Roudier F, et al. Vascularization of fetal cell suspension grafts in the excitotoxically lesioned adult rat thalamus. Brain Res Dev $1989 ; 48: 215-28$

50. Grabowski M, Christofferson RH, Brundin P, et al. Vascularization of fetal neocortical grafts implanted in brain infarcts in spontaneously hypertensive rats. Neuroscience $1992 ; 51: 673-82$

\section{TIRÉS À PART}

A. Gaillard

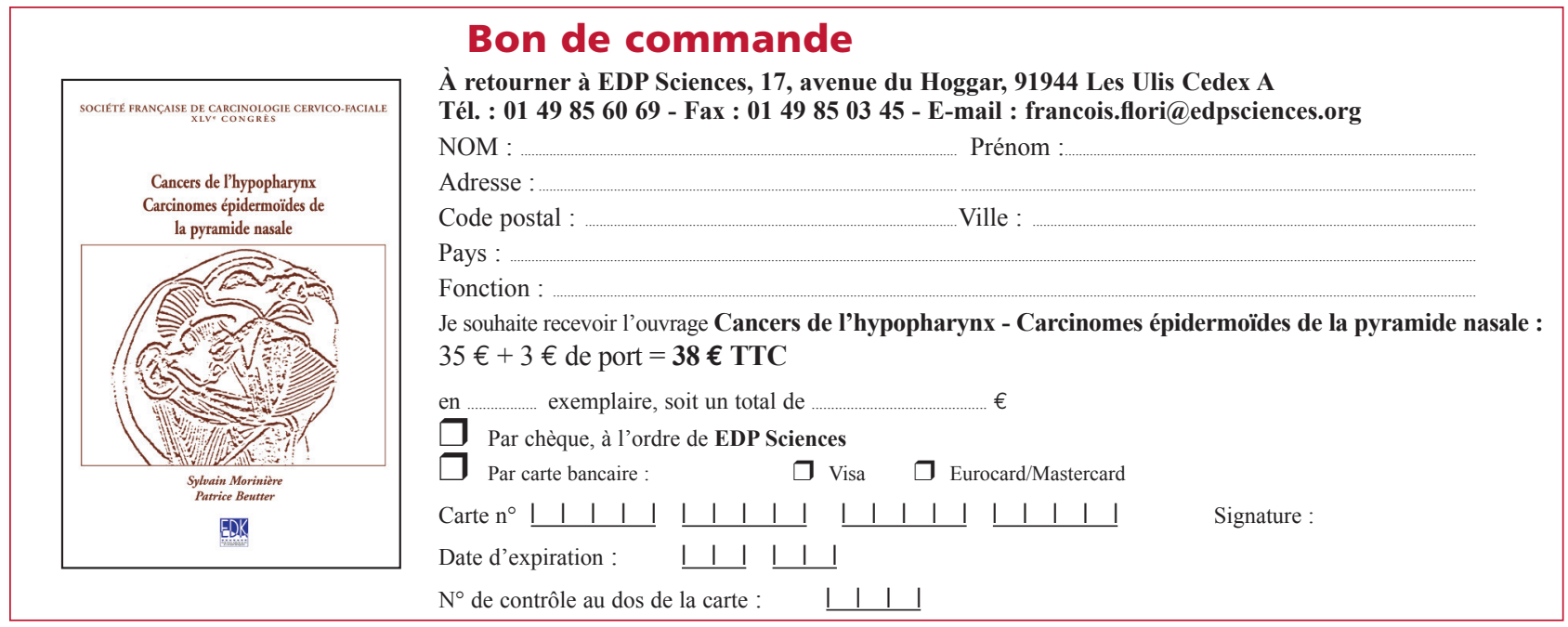

